\title{
Separation of high resolution spectra of the multiple star system UX Ari ${ }^{\star}$
}

\author{
V. Aarum Ulvås ${ }^{\star \star}$ and O. Engvold \\ Institute of Theoretical Astrophysics, PO Box 1029, Blindern, 0315 Oslo, Norway \\ Received 29 November 2002 / Accepted 7 January 2003

\begin{abstract}
This paper presents a technique for separation of individual components in composite stellar spectra. The technique is based on a comparison of residual depths of the component spectral lines to residual depths of the corresponding spectral lines of a single star of the same spectral class. We apply the technique to high resolution spectra of the RS CVn system UX Ari, where the spectral lines from all three components are well resolved. The deduced variations in the relative flux of the primary component agree well with simultaneously obtained photometric observations of UX Ari. The starspots causing the light curve variations and the facular areas causing the chromospheric emission seem to be separated by about $180^{\circ}$ in longitude on the surface of the primary component. We also find that the secondary component of UX Ari is subject to some chromospheric activity. Finally, some fundamental parameters of the third star in the spectrum are derived and discussed.
\end{abstract}

Key words. techniques: spectroscopic - stars: activity - binaries: spectroscopic - stars: imaging stars: individual: UX Ari - starspots

\section{Introduction}

Doppler imaging is a technique for reconstructing the distribution of a stellar surface characteristic (e.g. stellar surface temperature) by inverting a time sequence of high resolution, high signal-to-noise ratio spectral line flux profiles. Reviews of this technique are provided by e.g. Rice (1996) and Strassmeier (2001). Before the observed spectral line profiles can be inverted, one needs information on the unspotted photosphere of the target star. An inaccurate (incorrect) "unspotted spectrum", e.g. a stationary spectral feature in the line centre, may result in erroneous dark polar spots or a large polar facula. Usually one observes a single star of the same spectral type and luminosity class to get a correct input model atmosphere for the inversion. Since stellar activity depends on stellar rotation rate, and Doppler imaging needs a substantial rotational velocity, a large number of interesting cases belong to a binary (or multiple) system. In these cases it is necessary to separate the individual components in the combined spectra of two or more stars before deriving the correct surface structure of the active star.

Send offprint requests to: V. Aarum Ulvås,

e-mail:v.a.ulvas@astro.uio.no

* Based on observations made with the Nordic Optical Telescope, operated on the island of La Palma jointly by Denmark, Finland, Iceland, Norway, and Sweden, in the Spanish Observatorio del Roque de los Muchachos of the Instituto de Astrofisica de Canarias.

$\star \star$ Visiting Astronomer, Kitt Peak National Observatory, National Optical Astronomy Observatory, which is operated by the Association of Universities for Research in Astronomy, Inc. (AURA) under cooperative agreement with the National Science Foundation.
One approach could imply a simultaneous inversion of two or more stars. Alternatively, one may separate the individual spectra beforehand and subsequently treat the active component as a single star. Since this has not, to our knowledge, been done systematically before, we have taken this second approach in our processing of spectral observations of the RS CVn system UX Ari.

UX Ari (HD 21242) is a triple-lined system (Duemmler \& Aarum 2001, and references therein) where the two main components constitute a RS CVn-type binary. The spectral class of the primary component is $\mathrm{K} 0 \mathrm{IV}$, while the secondary component is a G5 V star (Carlos \& Popper 1971). UX Ari is listed in the catalogue of chromospherically active binary stars (CABS 28; Strassmeier et al. 1993) and in the Hipparcos catalogue (HIP 16042; ESA 1997).

In this paper, we describe the technique that has been used in separating the individual spectrum of the active primary component of UX Ari. As part of this process, we have investigated the chromospheric activity level of the secondary component as well as some fundamental characteristics of the third star in the spectrum. Finally, we present an analysis of the spectral flux variation and the chromospheric activity of the primary component.

Having separated the spectrum of the active primary component of UX Ari, we wish to apply the Doppler imaging technique to the observed spectra in order to reconstruct the temperature distribution on the surface of the active $\mathrm{K}$ star. A successful application of the Doppler imaging technique requires knowledge about the physical conditions of the 


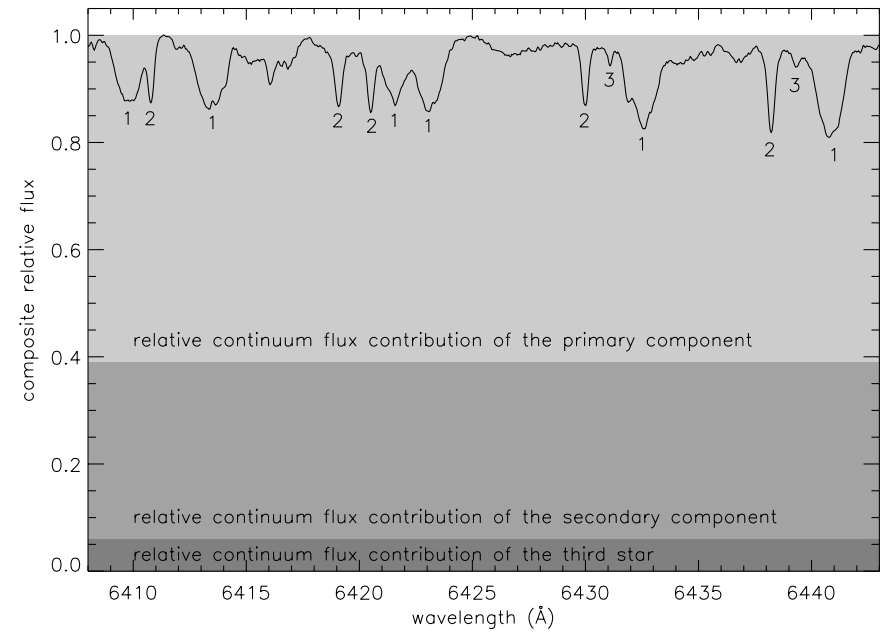

Fig. 1. The composite UX Ari spectrum from $6408 \AA$ to $6443 \AA$. Spectral lines of the primary K0 IV component, the secondary G5 V component and the third star are marked "1", "2" and "3", respectively. The relative continuum flux contributions of the three stars are indicated by the shaded regions.

unspotted stellar photosphere. Contemporary as well as long term photometry is an indispensable requirement for such a determination. Long term photometry of UX Ari has been published by Aarum Ulvås \& Henry (2003). The result from Doppler imaging and determination of the physical conditions of the unspotted photosphere will be described in some detail by Aarum Ulvås et al. (in prep.).

\section{Method}

The observed spectrum of a multiple star system is a composite of two or more spectral components. In this section we describe a technique for separating the various components of the composite spectrum of the UX Ari system. The technique can be applied to high resolution spectra of multiple stellar systems for separation of their individual spectral components.

As an illustration, Fig. 1 shows one observation of the composite UX Ari spectrum where spectral lines from the three components are well resolved and their relative contributions to the total flux are indicated.

\subsection{Description of the procedure}

The method assumes that, beside the primary stellar component, the spectral type and luminosity class of the secondary component as well as other, additional components can be reasonably well established. Besides the use of theoretical spectra calculated from standard model parameters, a more common approach is to secure spectra of a nearby, single star as close as possible to the spectral type and luminosity class in question. The spectra of such reference stars should preferably be observed close in time and with the same instrument as the multiple system itself. Since the reference stars are usually slow rotators compared to the corresponding stars of the multiple system, the line profiles of the reference spectrum are broadened by a simple convolution technique to mimic the proper rotational broadening, as explained in more detail in the following.

By comparing the observed depths of spectral lines belonging to the secondary and higher components in a composite stellar spectrum to the depths in the corresponding reference spectra, one may derive their relative contribution in the composite spectrum and thereby separate the spectral flux components.

In a $n$-component, normalized composite spectrum, we define the relative continuum flux level (or contribution) of component $i, \eta_{i}$, as follows:

$\eta_{i} \equiv \frac{\mathcal{F}_{i, \mathrm{c}}}{\sum_{j=1}^{n} \mathcal{F}_{j, \mathrm{c}}}$,

where $\mathcal{F}_{i, \mathrm{c}}$ is the continuum flux of component $i$. From the definition in Eq. (1) we see that the sum of all relative continuum flux levels in a composite spectrum equals 1 :

$\sum_{i=1}^{n} \eta_{i}=1$.

For a given multiple star system, the relative continuum flux levels can vary both with time and wavelength. If the system is eclipsing and/or at least one of the components is a variable star, $\eta_{i}$ will vary with time, i.e. with orbital phase. If the components of the star system have different temperatures, $\eta_{i}$ will vary with wavelength.

By applying the spectrum separation technique to the composite UX Ari spectrum, our aim has been to subtract the flux contributions of the secondary component and the third star, thereby isolating the spectrum of the K0 IV spotted primary component. In this process we derived the relative continuum flux levels of all three stars visible in the spectrum as functions of wavelength and time.

The spectrum of the secondary component was obtained by observing two reference stars, as described in Sect. 3.2. The reference star spectra were subsequently spun up to match the $v \sin i$ of the UX Ari secondary component. For a given UX Ari observation, the corresponding spun-up reference spectrum was scaled by an appropriate scale factor to reproduce the observed spectral line depth of the secondary component. The spun-up and scaled reference spectrum was finally subtracted from the observed UX Ari spectrum to yield a UX Ari spectrum without the flux contribution of the secondary component. The procedure was repeated for all observed UX Ari spectra, and all observations required slightly different scale factors. The set of appropriate scale factors was interpreted to represent the relative continuum flux level of the secondary component, $\eta_{2}$, as a function of wavelength (echelle spectral order) and time (orbital phase). The variation of $\eta_{2}$ with wavelength was interpreted to be caused by the different temperatures of the three stellar system components. The variation with time was interpreted to be caused by the continuum flux of the primary component varying due to the presence of starspots on its surface.

The spectral flux contribution of the third star in the spectrum was removed in the same way. The only difference was that it was not necessary to broaden the spectral lines of the 
reference star to match the rotational velocity of the third star, which means that the latter is not a fast rotator.

Once the spectral flux contributions of the secondary component and the third star were subtracted, we had obtained the continuum flux ratios $\eta_{2}$ and $\eta_{3}$ as functions of wavelength and time. From Eq. (2) one gets

$\eta_{1}=1-\eta_{2}-\eta_{3}$

which could be used to calculate $\eta_{1}$. However, the weaker spectral lines of the third star resulted in fewer measurements of $\eta_{3}$ than of $\eta_{2}$. In order to make use of all the measurements of $\eta_{2}$ in the calculations of $\eta_{1}$, we reformulated Eq. (3) to become

$\eta_{1}=1-\eta_{2}\left(1+\frac{\eta_{3}}{\eta_{2}}\right)=1-\eta_{2}\left(1+\frac{\mathcal{F}_{3, \mathrm{c}}}{\mathcal{F}_{2, \mathrm{c}}}\right)$.

Furthermore, by assuming that $\mathcal{F}_{3, \mathrm{c}} / \mathcal{F}_{2, \mathrm{c}}$ is constant in time, we could calculate $\mathcal{F}_{3, \mathrm{c}} / \mathcal{F}_{2, \mathrm{c}}$ at all points in wavelength and time where $\eta_{2}$ had been measured. This way, we were able to make use of all our measured values of $\eta_{2}$ to derive $\eta_{1}$ using Eq. (4) The assumption that $\mathcal{F}_{3, \mathrm{c}} / \mathcal{F}_{2, \mathrm{c}}$ is constant in time is equivalent to the assumption that both the secondary component and the third star are inactive. Further details on the calculations of $\mathcal{F}_{3, \mathrm{c}} / \mathcal{F}_{2, \mathrm{c}}$ are given in Sect. 3.4.

From Eq. (1) we see that

$\eta_{1} \equiv \frac{\mathcal{F}_{1, \mathrm{c}}}{\mathcal{F}_{1, \mathrm{c}}+\mathcal{F}_{2, \mathrm{c}}+\mathcal{F}_{3, \mathrm{c}}}$

In this expression, $\mathcal{F}_{1, \mathrm{c}}$, which varies with time, occurs in both the numerator and the denominator. To display the variation of $\eta_{1}$ more clearly, we introduce

$\eta_{1}^{\prime} \equiv \frac{\mathcal{F}_{1, \mathrm{c}}}{\mathcal{F}_{2, \mathrm{c}}+\mathcal{F}_{3, \mathrm{c}}}=\frac{1}{\frac{1}{\eta_{1}}-1}$.

In Eq. (6), the variable term occurs only in the numerator, and thus the variation of the whole expression will be more clearly visible. In order to make a straightforward comparison between the relative flux of the primary component and observed $V$ band photometry of UX Ari, we decided to convert $\eta_{1}^{\prime}$ to

$m_{1}^{\prime} \equiv-2.5 \log \eta_{1}^{\prime}$,

which yields a relative photometric variation of the primary star. For example, $m_{1}^{\prime}=-0.6$ means that the primary is $0.6 \mathrm{mag}$ brighter than the other two stars together.

\subsection{Practical considerations and limitations}

\subsubsection{Choice of reference star}

The reference star must fulfill several requirements to be suitable for the spectrum separation technique. It is required that:

- The reference star must be of closely the same spectral type and luminosity class as the component to be subtracted.

- The reference star must be recorded at the same time as the composite spectrum using the same instrument and the same instrument settings. The reason is to ensure that the two spectra have the same wavelength resolution and wavelength coverage.
- Since the reference star is to be observed whenever the multiple star system is observed, it should be an inactive star to ensure its spectrum does not vary.

- When the reference star is a slow rotator, its spectrum must be broadened to the same $v \sin i$ as the composite spectrum component to ensure that the spectral lines get the correct width (FWHM).

- Finally, the reference spectrum must be scaled to bring its spectral lines to the same residual line depths as the spectral lines of component $i$ (the component to be subtracted). The scale factor is the relative continuum flux contribution of component $i, \eta_{i}$.

\subsubsection{Constraints on the secondary component}

The method assumes that only the primary component is active and variable; the continuum levels of the other spectral components are assumed to be constant in time. In the event that one or more of the other components should be affected by some activity, it is essential that this activity does not influence the residual depth of the lines used for separation of the spectral fluxes.

We check whether or not these constraints are fulfilled for the G-type secondary component of UX Ari in Sect. 3.3.2.

\section{Separation of the three spectral flux components of UX Ari}

The UX Ari spectrum includes spectral lines from three components (see Fig. 1). The two brightest components constitute the RS CVn binary system. The third star in the spectrum was first mentioned by McAlister et al. (1987).

Duemmler \& Aarum (2001) published radial velocity measurements of the RS CVn system and concluded that there is a third body in the system causing an acceleration of the motion of the RS CVn binary. They also published radial velocity measurements of the third star in the spectrum covering 14 years and concluded that the apparent third component is not influencing the motion of the RS CVn binary, even though its own radial velocity is changing. Duemmler \& Aarum concluded that the third star just happens to lie in the line of sight of the RS CVn binary, and that it does not belong to the system.

\subsection{Observations and reduction of composite spectra}

The database of this study consists of 124 individual high resolution, high signal-to-noise ratio spectra that were obtained for Doppler imaging purposes (Aarum Ulvås et al., in prep.) in the course of five observing seasons.

The observations cover two wavelength regions:

1. The red wavelength region covers wavelengths from about $6000 \AA$ to about $7000 \AA$. This region was used by Duemmler \& Aarum (2001) for radial velocity measurements, and it contains the spectral lines to be used for Doppler imaging (Aarum Ulvås et al., in prep.).

2. The blue wavelength region covers the CaII $H \& K$ lines at $3969 \AA$ and $3934 \AA$, respectively. This region was 
Table 1. The number of spectra obtained of each target in each wavelength region in each observing season contained in the spectral database.

\begin{tabular}{lcrrrrrrrrr}
\hline \hline & \multicolumn{3}{c}{1995 Dec. } & \multicolumn{2}{c}{1996 Nov./Dec. } & \multicolumn{2}{c}{1999 Jan. } & \multicolumn{2}{c}{ 1999 Feb. } & \multicolumn{2}{c}{2000 Jan. } \\
Target & $6400 \AA$ & $3900 \AA$ & $6400 \AA$ & $3900 \AA$ & $6400 \AA$ & $3900 \AA$ & $6400 \AA$ & $3900 \AA$ & $6400 \AA$ & $3900 \AA$ \\
\hline UX Ari & 5 & - & 10 & - & 64 & 8 & 23 & 8 & 22 & - \\
HD 20630 & - & - & - & - & - & - & - & - & 7 & 7 \\
HD 23565 & - & - & 1 & - & - & - & - & - & - & - \\
HD 71148 & - & - & - & - & 1 & - & 1 & - & 1 & - \\
HD 76151 & - & - & - & - & - & - & - & - & 7 & 7 \\
\hline
\end{tabular}

Table 2. The wavelength resolution $(R=\lambda / \Delta \lambda)$ and range in signalto-noise ratios $(S / N)$ in the two observed wavelength regions for each observing season. The blue wavelength region was not observed in 1995 and 1996. The two $R$ values of 1999 February correspond to the first three nights/last five nights, respectively.

\begin{tabular}{lllll}
\hline \hline Season & $R$ & \multicolumn{2}{c}{$6400 \AA$ 政 } & \multicolumn{3}{c}{$3900 \AA$} \\
\hline 1995 Dec. & 86000 & $270-420$ & & $S / N$ \\
1996 Nov. & 71000 & $280-390$ & & \\
1999 Jan. & 36000 & $180-390$ & 31000 & $40-70$ \\
1999 Feb. & 86000 & $90-170$ & $91000 / 60000$ & $10-30$ \\
2000 Jan. & 48000 & $140-360$ & 39000 & $20-70$ \\
\hline
\end{tabular}

observed in order to study chromospheric activity of the UX Ari secondary component and its possible influence on the spectral line depths in the red wavelength region (see Sect. 3.3)

The spectral database was obtained using the following three combinations of telescope and spectrograph:

1. The high resolution SOFIN echelle spectrograph (Tuominen 1992) mounted at the Cassegrain focus of the 2.56 m Nordic Optical Telescope (NOT) on La Palma, Canary Islands, Spain.

2. The Sandiford Cassegrain Echelle Spectrometer mounted on the $2.1 \mathrm{~m}$ Otto Struve Telescope at McDonald Observatory, Texas, USA.

3. The Coudé CCD Spectrograph receiving light from the $0.9 \mathrm{~m}$ Coudé-Feed Telescope at Kitt Peak National Observatory, Arizona, USA.

The number of spectra of the five observing seasons is summarized in Table 1.

The reduction of the spectroscopic observations has been described by Duemmler \& Aarum (2001). Table 2 gives the wavelength resolution $(\lambda / \Delta \lambda)$ and the range of signal-to-noise ratios in both wavelength regions for each observing season. We adopted the projected equatorial rotational velocities $v \sin i=39 \mathrm{~km} \mathrm{~s}^{-1}$ for the primary component and $7.5 \mathrm{~km} \mathrm{~s}^{-1}$ for the smaller secondary component (Duemmler \& Aarum 2001). We also used the ephemerides of Duemmler \& Aarum, where orbital phase $\varphi=0$ corresponds to the time of maximum radial velocity of the primary component.

\subsection{Observations of reference stars}

For reference stars of the UX Ari secondary component we used the following two single, presumed inactive stars:

1995, 1996: HD 23565 (G5 V, $V=7.70 \mathrm{mag}$ )

1999, 2000: HD 71148 (G5 V, $V=6.30 \mathrm{mag}$ ).

The reference spectra were observed with the same instrument and instrument settings as the corresponding UX Ari spectra. The 1996 observation of HD 23565 was used in the analysis of both the 1995 and the 1996 spectra of UX Ari. The spectral lines of the reference star had to be artificially broadened before being used for subtracting the flux of the secondary component from the composite UX Ari spectrum. The "spin-up" was done for all data sets using a function in the 3A Software Package (Ilyin 1996) and linear limb-darkening coefficients from Al-Naimiy (1978).

Vogt \& Hatzes (1991) used a synthetic G5 V spectrum successfully to subtract the spectrum of the third star from the composite spectrum. We used observed and real spectra of HD 23565 and HD 71148 to achieve the same.

\subsection{Chromospheric activity in UX Ari}

\subsubsection{The primary component}

The observations of 1995 and 1996 include the Ca II 18662 line, which is an indicator of chromospheric activity. The observations of 1999 January and February contain UX Ari spectra in the blue wavelength region around the $\mathrm{Ca}$ II $\mathrm{H}$ and $\mathrm{K}$ lines. We were thus able to monitor the chromospheric emission of the primary component in 1995 December, 1996 November/December, 1999 January and 1999 February.

Figure 2 shows the Ca II 18662 line profiles of 1996 . The observation of UT date 1996 November 24 (HJD 2450411.56 ; middle profile in the left column) shows a central emission that is stronger than the emission observed by Gu et al. (2002) in 2000 September. Gu et al. classified their observation as a flarelike event, since the $\mathrm{He} \mathrm{I}_{3}$ line was in emission in the same exposure. Our observations do not include the $\mathrm{He} \mathrm{I}_{3}$ line, but since the emission was stronger in 1996 November than in 2000 September, we conclude that our observation of 1996 November 24 also shows a flare-like event.

Figure 3 shows the Ca II $\lambda 8662$ core emission of the primary as function of phase from 1995 and 1996. The core emission was calculated as the area between the core emission peak and a spline or polynomial representing the $\lambda 8662$ line 


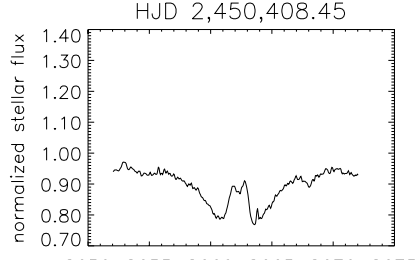

865086558660866586708675 wavelength $(\AA)$
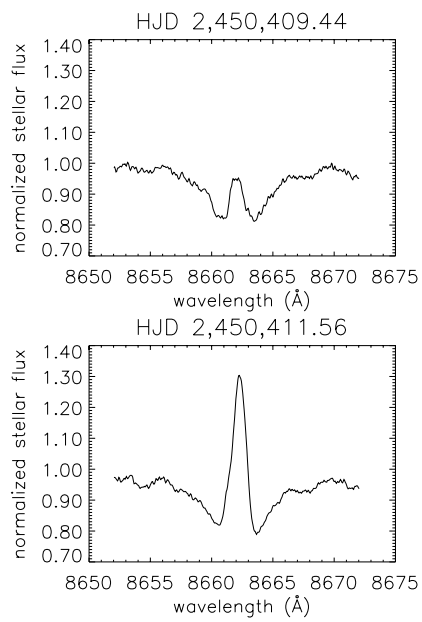

HJD 2,450,412.43

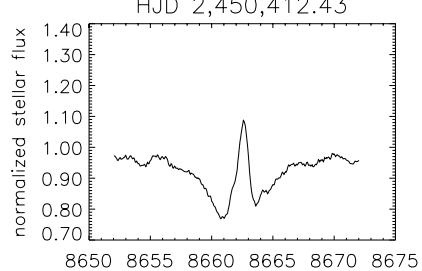

8650865586608665
wavelength $(\AA)$

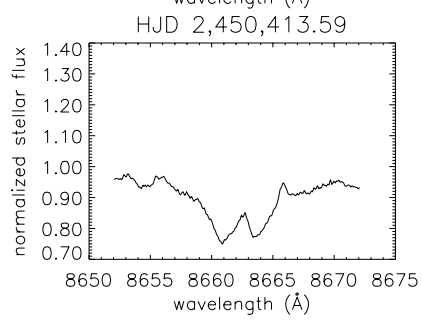

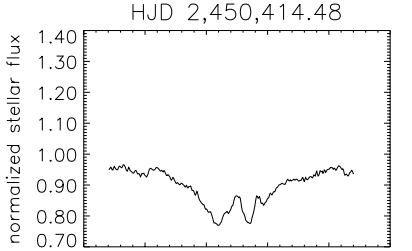

865086558660866586708675 wavelength $(\AA)$
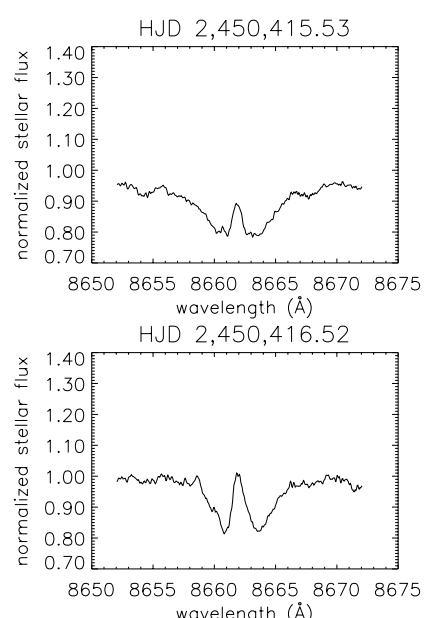

HJD 2,450,417.41

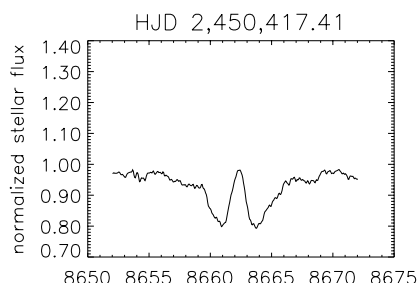

865086558660866586708675

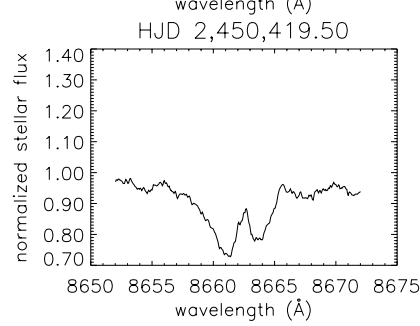

Fig. 2. The Ca II $\lambda 8662$ line profiles of the UX Ari primary component as observed in 1996 November/December.

without core emission. The errors in the figure reflect the average continuum normalization error in the $8662 \AA$ region of the 1995 and 1996 spectra of UX Ari. At phases near conjunction ( $\varphi \approx 0.25$ and $\varphi \approx 0.75$ ), the errors also reflect the additional emission from the secondary component.

Figure 4 shows the Ca II K core emission of the primary as function of phase in 1999 January and February. The emissions and their errors were calculated as explained above. The errors reflect the continuum normalization errors in the blue wavelength regions of the 1999 January and 1999 February spectra of UX Ari.

The Ca II chromospheric emission suggests that there is a persistent activity area around the stellar longitude corresponding to phase 0.2. Although we have no observations between 1996 December and 1999 January to confirm it, we assume that there could be an active region surviving more than two

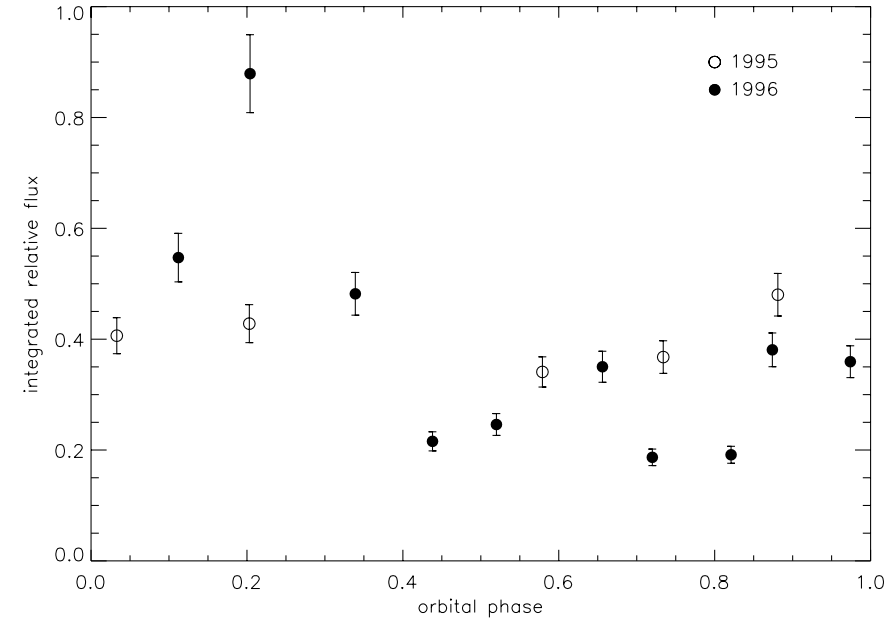

Fig. 3. The Ca II $\lambda 8662$ core emission of the UX Ari primary component as measured in 1995 December (open circles) and 1996 November/December (filled circles).

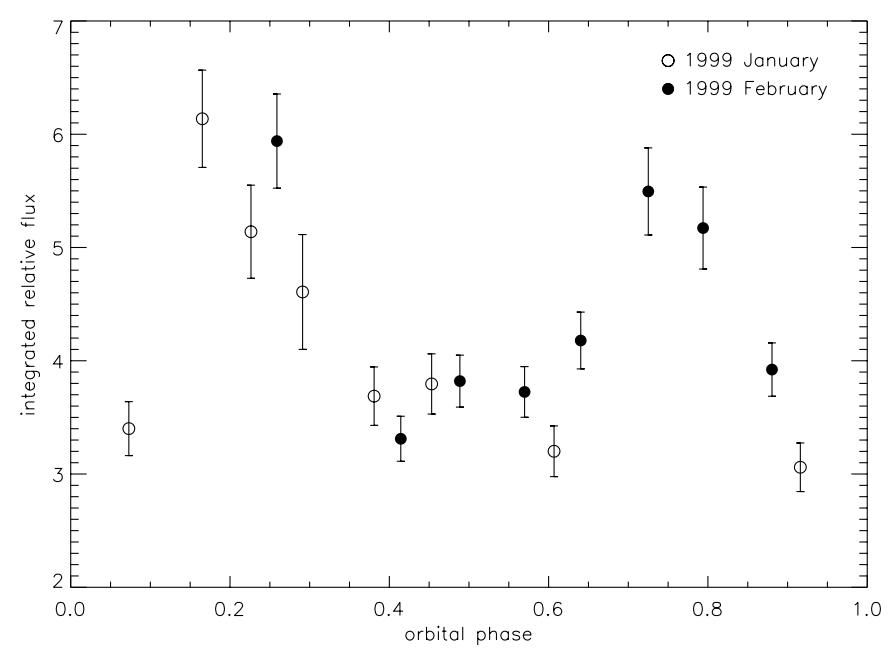

Fig. 4. The Ca II K core emission of the UX Ari primary component as measured in 1999 January (open circles) and February (filled circles).

years around this phase. In 1999 there seems to be another active longitude around $\varphi=0.7$.

\subsubsection{The secondary component}

As mentioned in Sect. 2.2.2, it is vital that any chromospheric activity of the secondary component does not influence the residual depth of the lines used for separation of the spectral fluxes. The way to check whether or not these constraints are fulfilled is to first establish whether the secondary component shows any chromospheric activity, and if so, to determine whether or not this activity influences the residual line depth in the spectral region of interest.

We have performed such a test of the G-type secondary component of UX Ari. Our conclusion is that although the secondary component does show a certain amount of chromospheric activity, this activity does not influence the average photospheric line depth. It is therefore feasible to derive the 

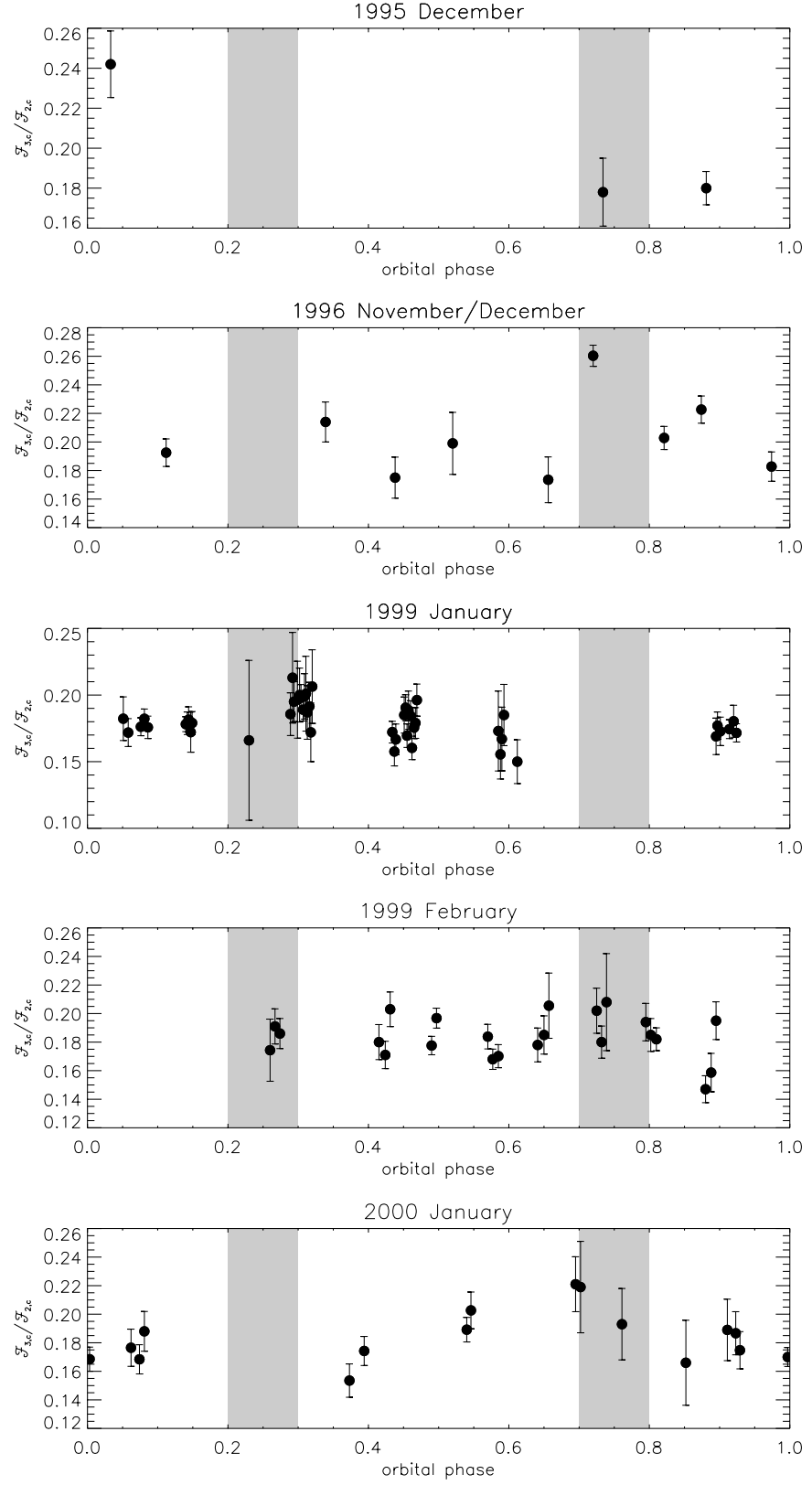

Fig. 5. The continuum flux ratio $\mathcal{F}_{3, \mathrm{c}} / \mathcal{F}_{2, \mathrm{c}}$ as function of orbital phase. The shaded regions indicate phases where the primary and secondary components are in conjunction.

flux ratio $\eta_{1}$ from Eq. (4). Further details on this test are given in Appendix A.

\subsection{The third star in the UX Ari spectrum}

The result of Sect. 3.3.2 is that the residual depths of the spectral lines of the secondary component are constant in time. Assuming that the same is the case for the third star in the spectrum, the flux ratio $\mathcal{F}_{3, \mathrm{c}} / \mathcal{F}_{2, \mathrm{c}}=\eta_{3} / \eta_{2}$ should be constant in time, and the wavelength dependence on $\mathcal{F}_{3, \mathrm{c}} / \mathcal{F}_{2, \mathrm{c}}$ should reflect the temperature difference between the two stars.

The deduced values of $\mathcal{F}_{3, \mathrm{c}} / \mathcal{F}_{2, \mathrm{c}}$ are plotted as function or orbital phase (i.e. averaged over wavelength) in Fig. 5. Figure 6 shows the flux ratio as function of wavelength (i.e. averaged

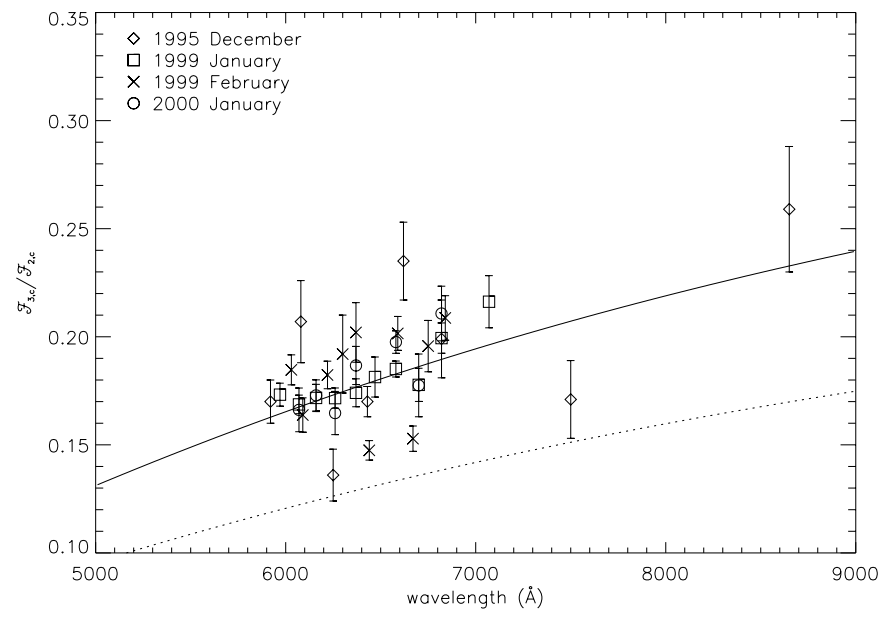

Fig. 6. The continuum flux ratio $\mathcal{F}_{3, \mathrm{c}} / \mathcal{F}_{2, \mathrm{c}}$ as function of wavelength. The orbital phases near conjunction (shaded regions in Fig. 5) were not included in these averages. The 1996 observations were not averaged, since the phase coverage was too poor, and the averaged flux ratio values deviated notably from the other seasons. The dotted and solid curves come from the least squares fit of the radius of the third star to the observed flux ratio; see the text for an explanation.

over orbital phase). Figure 6 clearly shows that the continuum flux of the third star in the spectrum is relatively stronger at longer wavelengths, indicating that the third star is cooler than the secondary component.

To use Eq. (4) to derive $\eta_{1}$ as function of wavelength and orbital phase, we need to calculate the continuum flux ratio $\mathcal{F}_{3, \mathrm{c}} / \mathcal{F}_{2, \mathrm{c}}$ as function of wavelength $\lambda$. The flux ratio may to a first approximation be described theoretically by the Planck functions and radii of the two stars:

$\frac{\mathcal{F}_{3, \mathrm{c}}}{\mathcal{F}_{2, \mathrm{c}}}=\frac{R_{3}^{2} B_{\lambda, 3}}{R_{2}^{2} B_{\lambda, 2}}$,

where $R_{2}, R_{3}, B_{\lambda, 2}$ and $B_{\lambda, 3}$ are the radii and Planck functions of the secondary component and the third star. When $R_{2}$ and $R_{3}$ and the temperatures $T_{2}$ and $T_{3}$ of the two stars are known, we use Eq. (8) to calculate the continuum flux ratio $\mathcal{F}_{3, \mathrm{c}} / \mathcal{F}_{2, \mathrm{c}}$. This flux ratio is then adopted into Eq. (4) to derive $\eta_{1}$.

From the literature we have $R_{2}=(1.11 \pm 0.08) R_{\odot}$ (Duemmler \& Aarum 2001) and $T_{2}=(5620 \pm 60) \mathrm{K}$, which is the average $T_{\text {eff }}$ from Gray (1992) and Cox (2000) for a G5 V star. The error in $T_{2}$ reflects the temperature difference between these two sources. Fabricius \& Makarov (2000) have determined $B-V=(1.19 \pm 0.06)$ for the third star, which yields $T_{3}=4400 \mathrm{~K}$ and $R_{3}=0.70 R_{\odot}$ (Gray 1992; Cox 2000), assuming that the third star is a main sequence star and that its light has not been influenced by interstellar extinction. The flux ratio from Eq. (8) using these stellar parameters is plotted as the dotted curve in Fig. 6.

The dotted curve in Fig. 6 is low compared to the observed flux ratios. A weighted least squares fit to the observed flux ratios implied $R_{3}=(0.83 \pm 0.02) R_{\odot}$ and $T_{3}=(4400 \pm 40) \mathrm{K}$. The derived temperature thus agrees with that derived from the $B-V$ measurements of Fabricius \& Makarov (2000). However, the radius is larger than the normal radius of a $\mathrm{K} 5$ main sequence star. 
Rather than assuming an unusual radius of the third star, we believe that it does not belong to the UX Ari system and that its relative flux is given by its distance from Earth, which is different from that of UX Ari. This result is in agreement with the conclusion of Duemmler \& Aarum (2001). The observed flux ratio implies a distance between Earth and the third star of $42 \mathrm{pc}$, or $84 \%$ of the distance between Earth and UX Ari (50.23 pc; ESA 1997).

Even though the third star in the spectrum does not seem to be gravitationally bound to the binary, Duemmler \& Aarum (2001) find that the centre-of-mass velocity of the binary changes systematically with time, indicating the presence of a fourth body. Duemmler \& Aarum find that the mass of this fourth body is at most $0.46 M_{\odot}$, which would yield a luminosity at most $\sim 10^{-3}$ times that of the primary component (Cox 2000). This is too faint to be detectable in the spectrum, and the fourth body was therefore not included in our theoretic modelling of the flux of the system.

\section{Spectral flux variations of the active primary component of UX Ari}

\subsection{Analysis}

We begin our analysis of the variation of the UX Ari primary component spectral flux by determining the wavelength dependence of the flux relative to the other two spectral components. The relative flux $m_{1}^{\prime}$ is defined in Eq. (7) and plotted against wavelength (i.e. averaged over orbital phase) in Fig. 7. The primary component is significantly brighter relative to the sum of the other two components at longer wavelengths. Since the third star in the spectrum is so faint, $m_{1}^{\prime}$ expresses basically the brightness of the primary component relative to the secondary component. The wavelength dependence of $m_{1}^{\prime}$ in Fig. 7 thus demonstrates that the primary component is cooler than the secondary component.

From a large number of high resolution spectra of UX Ari obtained during the five observing seasons we are now able to derive the photometric variations of the primary component of the system. These variations can be compared with regular photometry of the same system. In the following, we present plots of the relative flux $m_{1}^{\prime}$ against orbital phase and against heliocentric Julian date (HJD) for all our observing seasons. The plots are shown in Figs. 8-12.

As a reliability test of our measurements, we have compared our deduced values of $m_{1}^{\prime}$ to photometric observations of UX Ari in the $V$ band obtained at the same time as our spectroscopic observations (Aarum Ulvås \& Henry 2003). The activity of the primary component is the source of variation in $m_{1}^{\prime}$, but the same activity is generally accepted as the only source of variation in the brightness of the UX Ari system as a whole. The amplitude of variation in $m_{1}^{\prime}$ should therefore be the same as the amplitude of variation in observed $V$ photometry. The $V$ light curves of Aarum Ulvås \& Henry (2003) that correspond to our spectroscopic observations are also shown in Figs. 8-12.

Our measurements of $m_{1}^{\prime}$ fit in general quite well with the $V$ light curves. The best fits occur for the 1995 and 1996 data. We see a few deviating cases in our measurements, esp. from
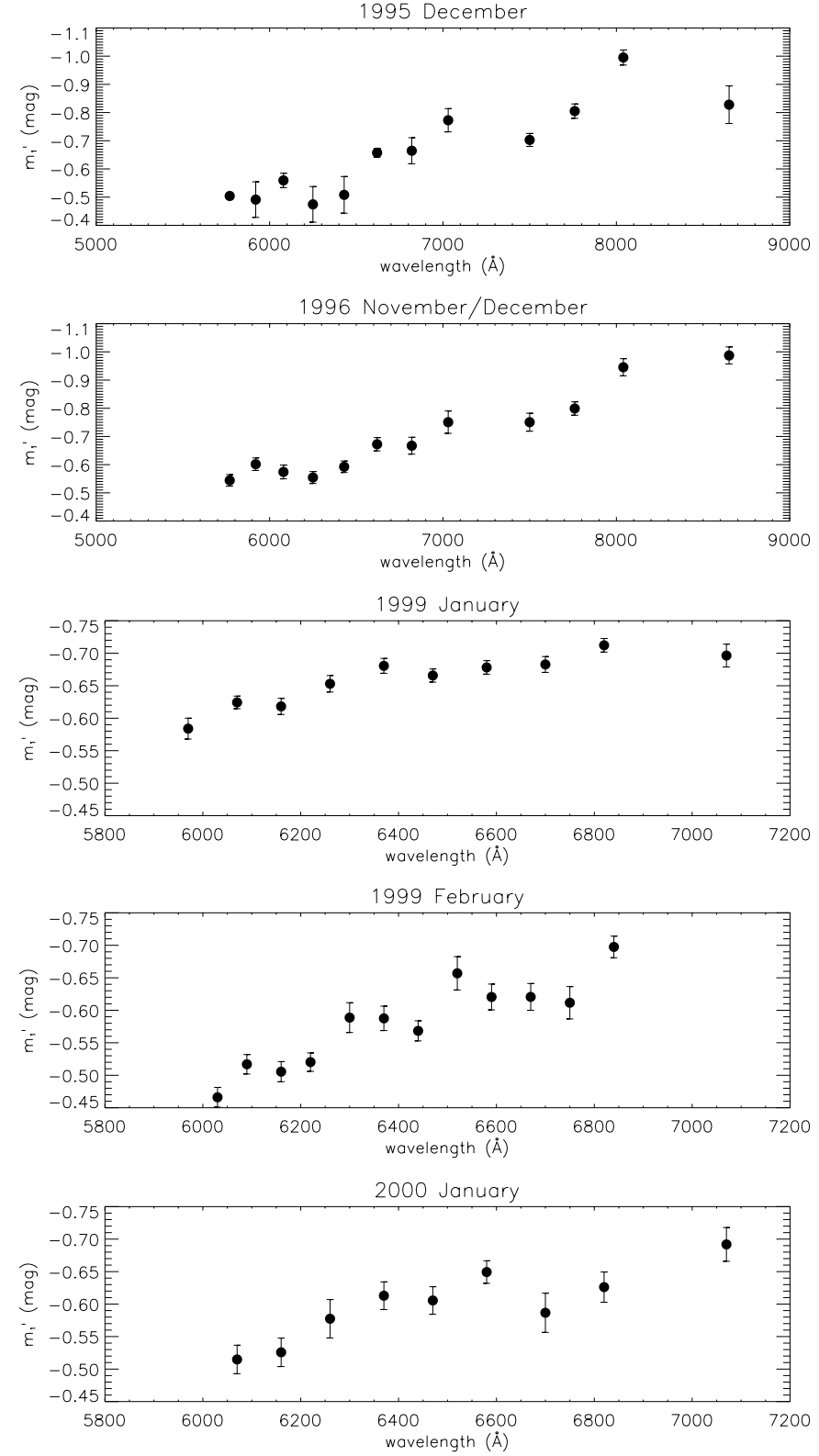

Fig. 7. The flux of the UX Ari primary component relative to the flux of the secondary component and the third star as function of wavelength from all our observations.

1999 February. We also see generally larger errors and larger spread near phases 0.25 and 0.75 , which are the phases where the primary and secondary components are in conjunction. The plots of $m_{1}^{\prime}$ and $V$ against HJD were made to illustrate the amount of photometric observations that were taken simultaneously with our spectroscopic observations, but also to check individual deviating measurements of $m_{1}^{\prime}$ against simultaneously obtained $V$ measurements. One such deviating $m_{1}^{\prime}$ measurement that stands out is the one of HJD 2 450 408.45, $\varphi=0.72$ (Fig. 9). One photometric measurement is taken during the same night, and although the $m_{1}^{\prime}$ flux deviates significantly from the photometric light curve, the photometric measurement of the same night does not. We therefore conclude that the $m_{1}^{\prime}$ measurement of that night must be erroneous. 


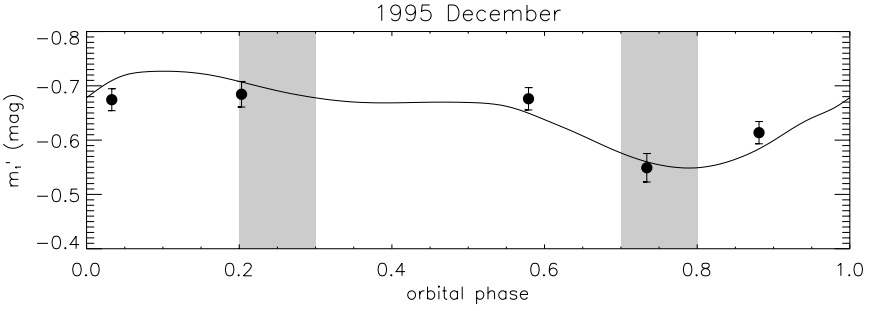

$1995 / 1996$ differential $\vee$ photometry
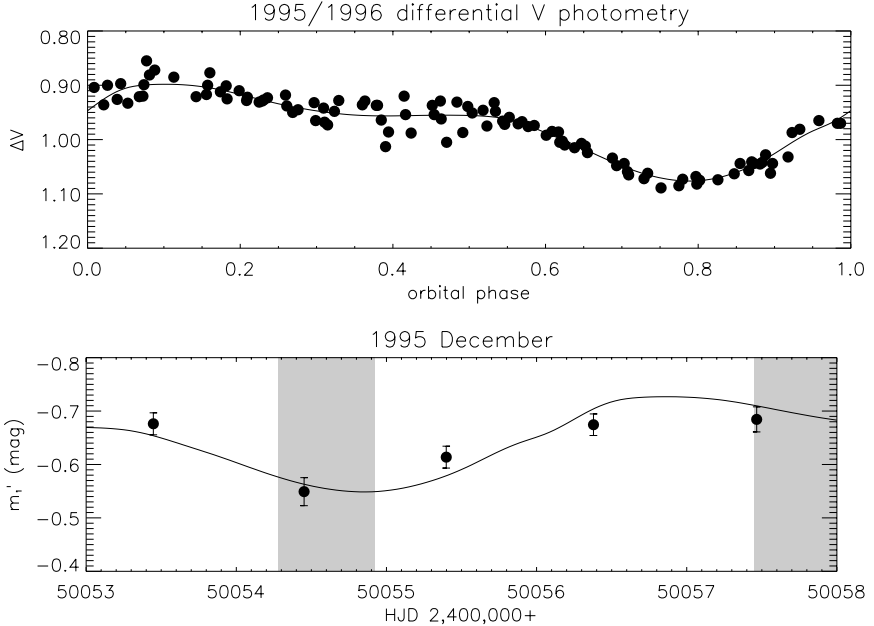

Differential $\vee$ photometry

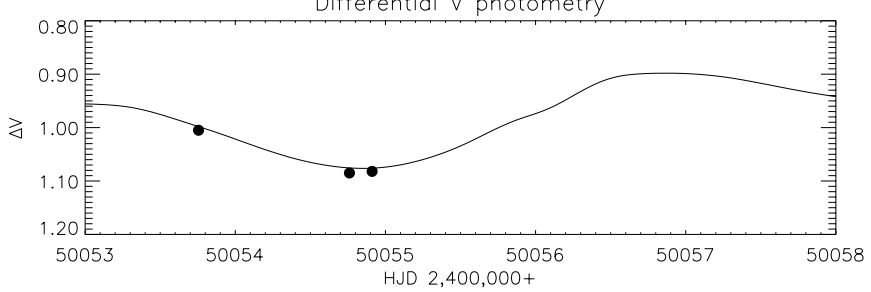

Fig. 8. Top: The relative flux of the primary component, $m_{1}^{\prime}$, as measured from our 1995 December observations against orbital phase. The shaded regions indicate phases where the primary and secondary components are in conjunction. The solid curve is a cubic spline fit to the photometric observations of Aarum Ulvås \& Henry (2003). Second from top: Differential $V$ photometry of UX Ari from the 1995/1996 observing season (Aarum Ulvås \& Henry 2003) as function of orbital phase. The solid curve is a cubic spline fit to the data. Third from top: $m_{1}^{\prime}$ as function of heliocentric Julian date (HJD) of observation. The shaded regions indicate times near conjunction. The solid curve is a cubic spline fit to the photometric observations of Aarum Ulvås \& Henry (2003). Bottom: The differential $V$ photometry by Aarum Ulvås \& Henry (2003) as function of HJD of observation. The solid curve is a cubic spline fit to the photometric observations.

\subsection{Comparison with chromospheric emission}

Also the relative flux $m_{1}^{\prime}$ and the photometric light curves have been compared with the chromospheric emission of UX Ari. The $V$ light curve of the 1995/1996 observing season (Fig. 8) shows a minimum at orbital phase 0.8 , whereas the 1995 December Ca II $\lambda 8662$ emission (Fig. 3) is virtually flat. The Ca II $\lambda 8662$ emission of 1996 November/December (Fig. 3) shows a strong peak at orbital phase 0.2 , which does not correspond to any notable signature in the photometric observations (Fig. 9). The light curve minimum of 1996/1997 occurs at orbital phase 0.7, but there is no peak in the Ca II $\lambda 8662$ emission

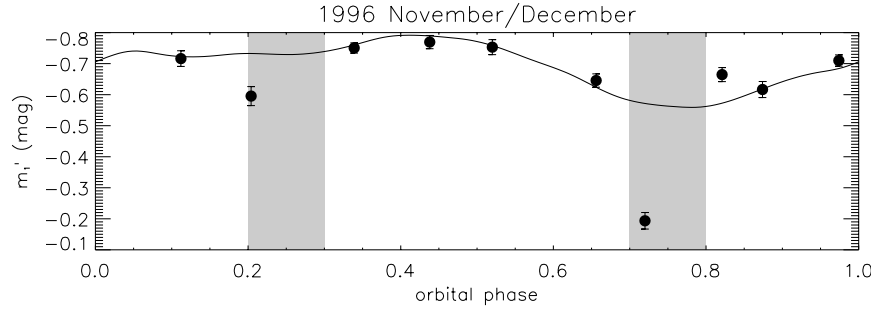

1996/1997 differential V photometry
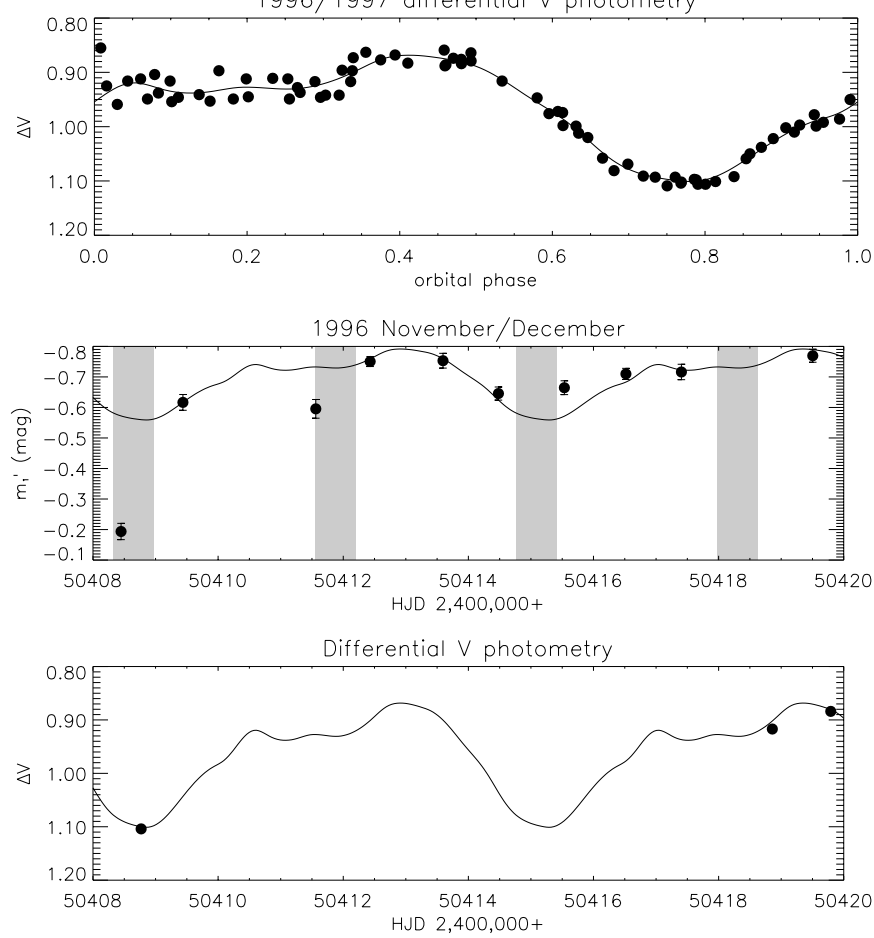

Fig. 9. The same as Fig. 8, but for the 1996 observations.

at this orbital phase. The Ca II K emission of 1999 January (Fig. 4) shows a strong peak at orbital phase 0.2, but there is no clear minimum neither in $m_{1}^{\prime}$ nor in the $V$ magnitude at this orbital phase (Fig. 10). In 1999 February, the Ca II K emission peaks at orbital phases 0.2 and 0.7 (Fig. 4). The $m_{1}^{\prime}$ measurements of 1999 February (Fig. 11) has a maximum at orbital phase 0.8 , although this lies within the orbital phase range of conjunction. There is a minimum in the $V$ light curve of the 1998/1999 observing season (Fig. 11) at orbital phase 0.7, but there is no clear light curve minimum at orbital phase 0.2.

A comparison of $m_{1}^{\prime}$ with Ca II emission shows no strong correlation, indicating that the variations in the $V$ light curves of UX Ari are predominantly caused by spots. The chromospheric emission does, however, influence the colour of the system (Aarum Ulvås \& Engvold 2003). Both the $V$ magnitude and the $\mathrm{Ca}$ II emission presented here show strong rotational modulation, but they are shifted in phase relative to each other. The spots causing the $V$ magnitude variations and the facular areas causing the $\mathrm{Ca}$ II emission seem to be separated by about $180^{\circ}$ in longitude. 

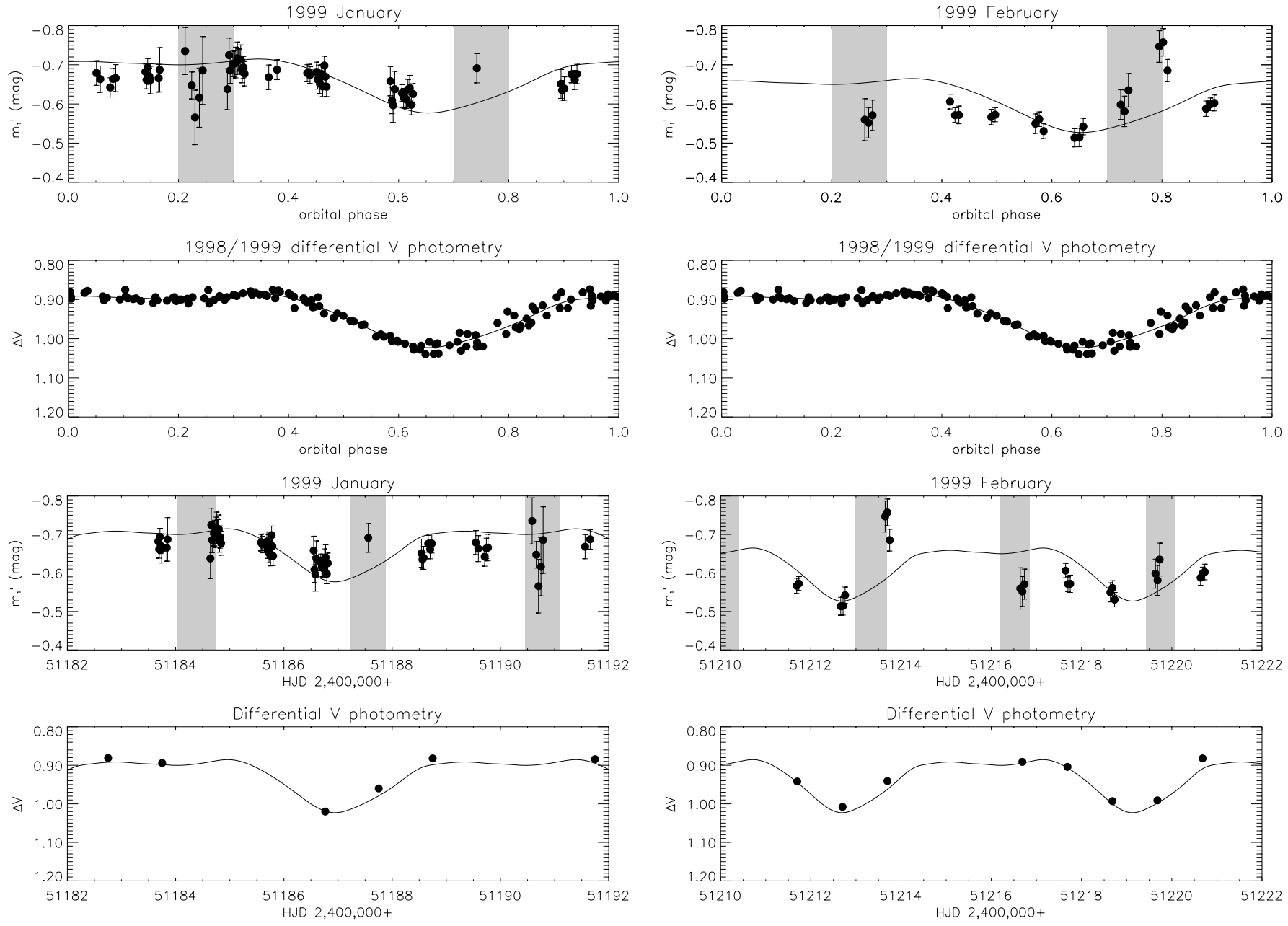

Fig. 10. The same as Fig. 8, but for the 1999 January observations.

\subsection{Discussion of errors and error sources}

Our technique is subject to errors and uncertainties of various kinds, and we consider the most important ones in the following.

The relative flux levels of the individual components are measured from the residual depths of the photospheric spectral lines of the same components. Any errors made when normalizing the spectra to the local continuum will result in erroneous depths of the spectral lines of all components. This will subsequently influence the measurements of the relative flux levels. Similarly, the relative flux level measurements will be influenced by any differences in spectral line shape or depth between the programme star and the reference star.

The relative flux levels are derived as functions of wavelength and orbital phase. In this paper, they are presented as either function of wavelength (and consequently averaged over orbital phase) or function of orbital phase (consequently averaged over wavelength). When averaging over orbital phase (Figs. 6 and 7), care has been taken to eliminate the phases close to conjunction in the average. When averaging over wavelength (Figs. 5 and $8-12$ ), the result is influenced by which points in wavelength are available for the average, since the relative flux levels vary systematically with wavelength.

Fig. 11. The same as Fig. 8, but for the 1999 February observations.

Despite the error sources, the relative flux of the primary component of UX Ari derived from our spectral data agrees well with the observed photometry of UX Ari (Figs. 8-12). The deviations from the observed $V$ light curves can be explained by the effect of conjunction between the primary component and the secondary component. The measurement errors produced by the separation technique seem to be larger than the general spread in the photometric observations.

\section{Conclusions}

In this paper, we have presented a technique for separation of the spectra of individual components in a triple-lined stellar spectrum. In principle, the method can be applied to composite stellar spectra made up of any number of components. We have applied this technique to observed spectra of the triplelined system UX Ari and compared our results to simultaneous photometry. Our main results are summarized below:

- We have separated the individual spectra of the active primary component of UX Ari. These spectra will be used in Doppler imaging studies of this star (Aarum Ulvås et al., in prep.). 


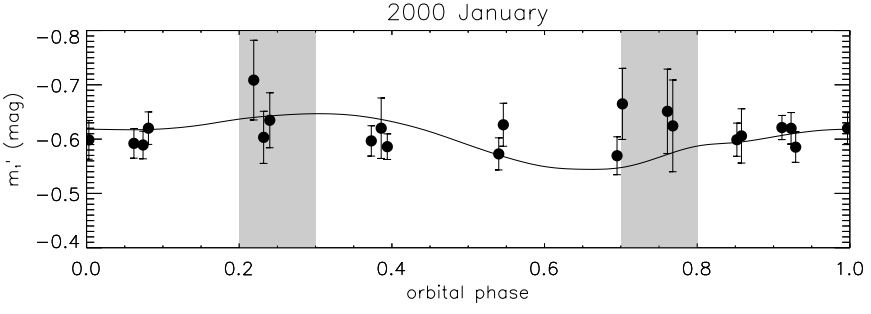

$1999 / 2000$ differential $\vee$ photometry
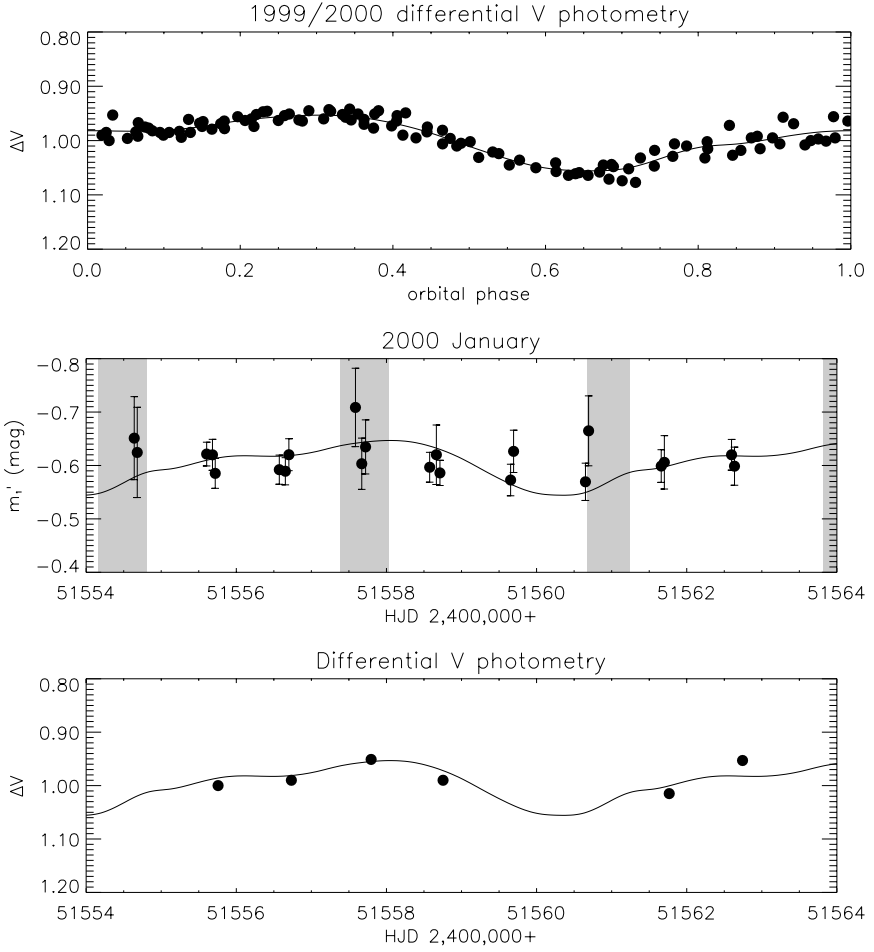

Fig. 12. The same as Fig. 8, but for the 2000 observations.

- The derived relative flux of the active primary component shows good agreement with simultaneous photometric observations of UX Ari.

- The separation technique does not function well near the orbital phases where the primary and secondary components are in conjunction. However, since the technique gives good results outside the phases of conjunction, the simultaneous photometric observations enable us to derive with confidence the relative flux of the primary component also at the phases of conjunction.

- The UX Ari primary component shows strong and varying chromospheric emission. The regions on the stellar surface that are responsible for this emission, seem to be separated by about $180^{\circ}$ from the spots that give rise to the $V$ magnitude variations.

- The secondary component also shows some chromospheric activity, but it could be shown that this activity does not influence the average residual depth of the photospheric spectral lines used for the separation of individual component spectra.

- The third star that is present in the composite spectrum, is not part of the UX Ari system. It is located at a distance from Earth that is different from the distance between Earth and UX Ari. Its effective temperature is $T_{\text {eff }}=(4400 \pm$ 40) $\mathrm{K}$.

Acknowledgements. This research has made use of the Simbad database, operated at CDS, Strasbourg, France. The main part of this work was supported by the Norwegian Research Council under project number 122520/431. VAU acknowledges financial support from Institute of Theoretical Astrophysics, University of Oslo, and support from WesternGeco.

\section{Appendix A: Chromospheric activity of the secondary component}

In search for possible chromospheric activity of the secondary component of UX Ari we used observations of UX Ari in the blue wavelength region covering 1.22 rotational periods and obtained in 1999 January. The opposite and changing spectral line shifts caused by the orbital motions of the two main components allowed us to separate their Ca II K line emissions, which subsequently could tell if the secondary star showed any sign of activity variation. Figure A.1 shows the Ca II K core emission of the secondary component from 1999 January. The emission and its errors were derived as explained in Sect. 3.3.1. The errors reflect the uncertainties in the continuum normalization in the blue wavelength region of the 1999 January spectra of UX Ari.

The Ca II K core emission of the secondary component is substantial and varies noticeably, according to Fig. A.1. Chromospheric emission from the secondary component in the Ca II $\mathrm{H}$ line has previously been reported by Huenemoerder et al. (1989). The spectrum separation procedure assumes that the secondary component is inactive, which more specifically means that its photospheric spectral line depths do not vary. Given the varying chromospheric Ca II $\mathrm{K}$ core emission in Fig. A.1, does the varying activity level influence the residual photospheric spectral line depth in the wavelength region used for Doppler imaging?

Since some chromospheric variability could be noticed, the next step was to check, by means of properly chosen

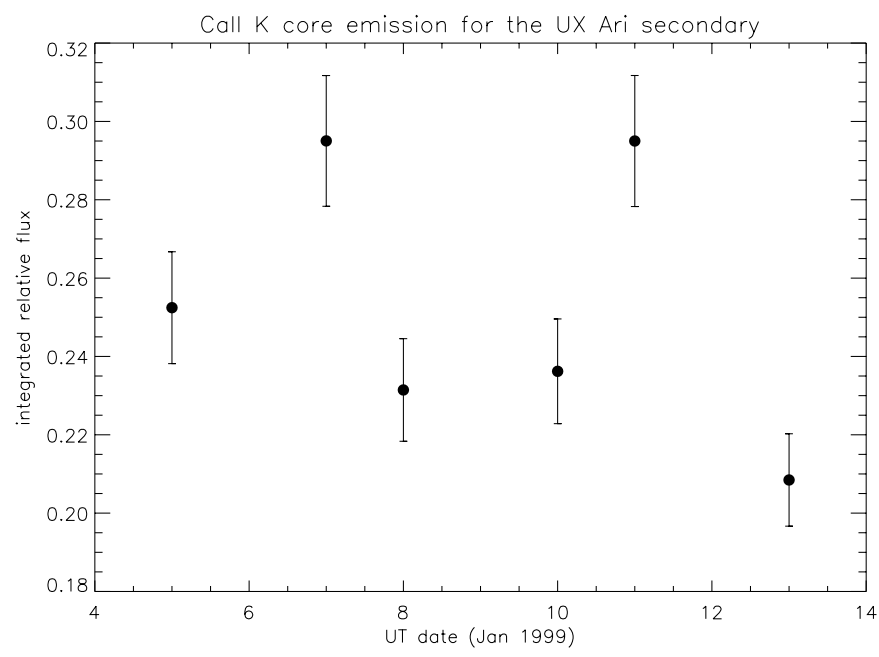

Fig. A.1. The Ca II K core emission for the UX Ari secondary component measured from the 1999 January spectra. 


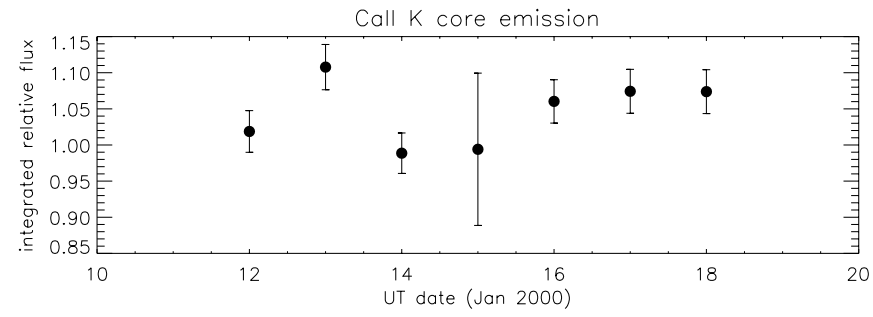

Average residual line depth

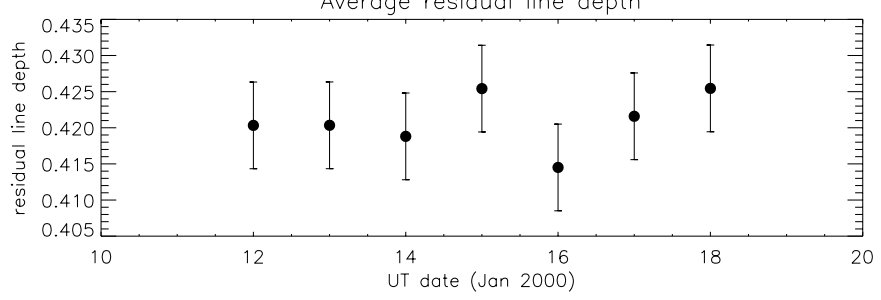

Fig. A.2. The Ca II K core emission (top) and the average photospheric residual line depth (bottom) for HD 20630.

chromospheric activity reference stars, whether this level of chromospheric activity could lead to any measurable changes in the strengths and depths of the lines used for relative flux separation. We made observations of two single, chromospherically active stars, HD 20630 and HD 76151, in both the red and the blue wavelength region. The objective was to compare the Ca II K core emission and the average (over wavelength) photospheric line depth, and to see if any corresponding variations in the photospheric lines could be detected.

Figure A.2 shows the results for HD 20630. The top panel shows the measured Ca II K core emission for each day of observation. The errors in the top panel were derived in the same way as the errors in Figs. 3, 4 and A.1, and reflect the continuum normalization errors in the blue spectra of HD 20630. The dramatically larger error on UT date Jan. 15 (Fig. A.2) comes from poor seeing during that exposure leading to stronger noise and larger uncertainties in determining the continuum level of the spectrum. The bottom panel shows the average residual depth of 10 spectral lines in the wavelength interval ( $6390 \AA, 6450 \AA$ ). The residual depth of an individual absorption line profile was determined by fitting a Gaussian profile to the absorption line profile and recording the residual depth of the Gaussian. The errors in the bottom panel come from errors in Gaussian profile fitting and in continuum normalization. The strongest variation in Ca II $\mathrm{K}$ core emission occurs from UT dates Jan. 12 to Jan. 14. During the same period we see no significant change in average photospheric line depth.

Figure A.3 of HD 76151 corresponds to Fig. A.2 of HD 20630. In the bottom panel, the average residual line depth of UT date Jan. 14 is missing, because the spectrograph was out of focus during this particular exposure. HD 76151 shows stronger variations in the chromospheric Ca II $\mathrm{K}$ core emission than HD 20630. However, in both cases we detect no significant variation in the average photospheric line depth.
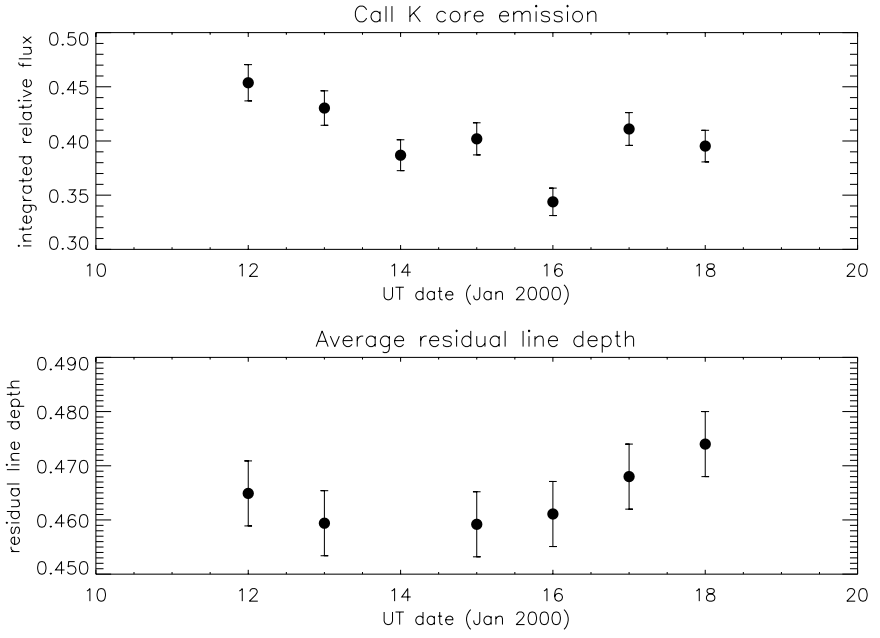

Fig. A.3. The Ca II K core emission (top) and the average photospheric residual line depth (bottom) for HD 76151.

In conclusion, although the UX Ari secondary component does show a certain level of chromospheric activity, this activity does not appear to influence the average photospheric line depth. Therefore, it seems safe to calculate the relative continuum flux level of the primary component, $\eta_{1}$, using Eq. (4).

\section{References}

Aarum Ulvås, V., \& Engvold, O. 2003, A\&A, 399, L11

Aarum Ulvås, V., \& Henry, G. W. 2003, A\&A, 402, 1033

Al-Naimiy, H. M. 1978, Ap\&SS, 53, 181

Carlos, R., \& Popper, D. M. 1971, PASP, 83, 504

Cox, A. N., (ed.) 2000, Allen's Astrophysical Quantities (New York: Springer)

Duemmler, R., \& Aarum, V. 2001, A\&A, 370, 974

ESA. 1997, The Hipparcos and Tycho catalogues, vol. 1-17, ESASP-1200

Fabricius, C., \& Makarov, V. V. 2000, A\&A, 356, 141

Gray, D. 1992, The observation and analysis of stellar photospheres, 2nd ed. (Cambridge: Cambridge University Press)

Gu, S.-H., Tan, H.-S., Shan, H.-G., \& Zhang, F.-H. 2002, A\&A, 388, 889

Huenemoerder, D. P., Buzasi, D. L., \& Ramsey, L. W. 1989, AJ, 98, 1398

Ilyin, I. V. 1996, Acquisition, Archiving and Analysis (3A) Software Package - User's Manual, Observatory, University of Helsinki

McAlister, H. A., Hartkopf, W. I., \& Hutter, D. J. 1987, AJ, 93, 688

Rice, J. B. 1996, in Stellar Surface Structure, ed. K. G. Strassmeier, \& J. L. Linsky (Kluwer Academic Publishers), IAU Symp., 176, 19

Strassmeier, K. G. 2001, in PASPC, Vol. 223, Cool Stars, Stellar Systems and the Sun, Eleventh Cambridge Workshop, ed. R. J. García López, R. Rebolo, \& M. R. Zapatero Osorio, 271

Strassmeier, K. G., Hall, D. S., Fekel, F. C., \& Scheck, M. 1993, A\&AS, 100, 173

Tuominen, I. 1992, NOT News, 5, 15

Vogt, S. S., \& Hatzes, A. P. 1991, in The Sun and Cool Stars: activity, magnetism, dynamos, ed. I. Tuominen, D. Moss, \& G. Rüdiger (Springer-Verlag), IAU Coll., 130, 297 